\title{
Mediating Effects of Value Congruence on Relationships between Motivation and Organizational Commitment in Nonprofit Sport Organizations ${ }^{1}$
}

\author{
Wu xiaojun ${ }^{1}$ Zhu zhiquan ${ }^{2}$ Wu hongyv ${ }^{3}$ \\ 1, Human Resources, East China Institute of Technology, Nanchang 330013, China \\ ${ }^{2,}$ Research Department, East China Institute of Technology, Fuzhou 344000, China \\ ${ }^{3}$ School of Physical Education, East China Institute of Technology, Fuzhou 344000, \\ China
}

\begin{abstract}
The purpose of this paper is to measure the mediating role of Value Congruence in the relationship between motivation and organizational commitment in nonprofit sport organizations. An e-mail survey was conducted with a sample of 223 volunteers in 12 non-profit sport organizations in a Jiangxi Province of China. Findings supported the partial mediation role of Value Congruence. The values factor of motivation had a significant direct impact on organizational commitment, and value congruence partially mediated the relationship between values and organizational commitment. The study contributes to the understanding of volunteers' motivation and how it influences commitment toward non-profit sport organizations.
\end{abstract}

Keywords: Value Congruence, motivation, organizational commitment, Volunteers

\section{Introduction}

Nonprofit sport organization (NPSO) is one type of nonprofit organizations and is driven by values of unity to organizations. Nowadays NPSO has become a component part that cannot be substitute in sport organization structure, as demands of public sports public service of our country is growing rapidly and consequently. Considering this volunteer contribution to sport organizations, maintaining the volunteer base is one of the most significant tasks in non-profit sport organizations. This study integrated three important factors based on social exchange theory: volunteers' motivation, organizational commitment, and value congruence to understand the process of individuals' consistent contribution to volunteering in non-profit sport organizations.

The purpose of the present study was to examine the mediating role of value congruence in relationship between volunteers' motivation and organizational

\footnotetext{
${ }^{1}$ This work was supported by a grant from human social science Programs of Educational Commission of Jiangxi Province, China (Grant No. TY1201 )
} 
commitment in the context of non-profit sport organizations. Understanding a possible "causal" relationship may provide researchers and volunteer managers with vital new information about how to development organizational commitment, an important organizational outcome. Therefore, the following hypotheses are proposed:

H1. Value congruence mediates the relationship between measuring of volunteer motivation and organizational commitment.

H2. Value congruence mediates the relationship between understanding of volunteer motivation and organizational commitment.

$\mathrm{H} 3$. Value congruence mediates the relationship between social of volunteer motivation and organizational commitment.

H4. Value congruence mediates the relationship between improvement of volunteer motivation and organizational commitment.

\section{Method}

\subsection{Participants}

The sample included 223 participants (35.7 percent female and 64.3 percent male). Age ranged from 18 to 63 with the mean age of 34.0. The volunteers in these organizations consisted of all levels of continuous volunteers who offered their time and effort to an organization regularly over a period of time, longer than six months.

\subsection{Measures}

Three primary scales and demographic questions formed the survey battery. First, the organizational commitment scale (OCS) developed by Meyer and Allenwas used to measure organizational commitment. Seven items of the OCS were adjusted to reflect the volunteer context of non-profit sport organizations.
Second, Clary et al.'s volunteer functions inventory (VFI) was used to measure volunteer motivation (the 20 items were modified to better represent volunteer contexts). Four dimensions of the VFI: belief, understanding, social, and enhancement were included in the questionnaire. The VFI is one of the few measurement scales of volunteer motivation to undergo extensive testing by volunteers themselves. Finally, five items described general value congruence employing the Job Diagnostic Survey by Tan xiaohong. Tan xiaohong's value congruence scale has been used in a number of organizational studies, including volunteer studies in the context of non-profit organizations. All responses to the respective scales (seven-point Likert type) ranged from strongly disagree (1) to strongly agree (7). The survey also included four questions assessing basic demographic questions (sex, age, marital status, and level of education) for the purpose of developing a respondent profile. The final instrument included 36 items. Table I presents the items included in the study's questionnaire.

\subsection{Data Analysis}

Structural equation modeling (SEM) was conducted to test the H1-H4 using the statistics program LISREL 7. Confirmatory factor analysis (CFA) in SEM evaluated the meaningfulness of latent variables and their indicators. For the items selected to represent the various constructs of the both independent and dependent variables, the internal consistency estimate, Cronbach's a, were then computed. The SEM allowed a test of the entire system of relationships between volunteer motivation, value congruence, and organizational commitment constructs. Baron and Kenny suggested that before testing for a mediating effect, there must be a significant relationship between the latent 
predictor variables and the latent outcome variables. Thus, after the fit of the measurement model was determined using CFA, the hypotheses were tested using structural model tests. Three different structural models- one full and two nested models - were tested and compared.

Model I examined the direct path in the model without a mediator. Model II examined a full model that included all direct and indirect paths. Finally, Model III examined a mediation model without a direct path. For the model testing, it was assumed that a model had a latent predictor variable, a hypothesized latent mediator variable, and a latent outcome variable Model I was first tested to show that the direct path from volunteer motivation to organizational commitment was significant. A $\chi^{2}$-difference test was then performed to examine if the full model was significantly different than the two nested models. That is, Model I and Model III were examined as a subset of Model II including all the effects by subtracting the $\chi^{2}$ and degrees of freedom.

\section{Results}

\subsection{Measurement Model}

The measurement model included 22 items comprising the six constructs covering organizational commitment, value congruence, and four volunteer motivation constructs. The CFI (0.92), the NNFI (0.91), and the IFI (0.92) indicated an adequate fit of the data given that each exceeded the minimum cutoff of 0.90 . The RMR value (0.07) was acceptable with there commended criteria of 0.08 for a reasonable fit. Although the RMSEA (0.084) slightly exceeded the recommended criteria of 0.08 for a reasonable fit, according to Kenny and
McCoach's study, these types off it indices may degrade when models enclose large numbers of variables. Thus, based on Kenny and McCoach's suggestion and that other fit indices met their criteria, and all t-values were statistically significant (the minimum tvalue was 7.14), the CFA results pointed out that the measurement model achieved a reasonable fit to the data. The results provide evidence of factorial validity and convergent validity of the study's measurement. The reliabilities meet the minimum level $(0.70)$ recommended by Nunnally and Bernstein. All of these correlations were significant, but the highest correlation of 0.62 between values and understanding resulted in a shared variance of only 41.4 percent. The results thus suggested that the four motivational dimensions were sufficiently distinct from each other and that they represented unique constructs.

\subsection{Testing structural model}

The SEM results revealed that Model I had a marginally acceptable fit to the data (RMSEA $=0.086, \mathrm{CFI}=0.90, \mathrm{NNFI}=0.91$, $\mathrm{IFI}=0.91$, and $\mathrm{RMR}=0.072$ ). However, most of the relationships were not significant, except the relationship between values and organizational commitment was significant. Baron and Kenny suggested that there must be a significant relationship between the predictor variable and the outcome variable to test for a mediating effect. Therefore, the stepwise variable selection with backward elimination was used by choosing a variable with the lowest $t$ value and eliminating it from the model one at a time. The finally selected model included only one volunteer motivation factor, values $\left(\chi^{2}=61.50, \mathrm{df}=18, \mathrm{p}<0.01\right.$, RMSEA $=0.15, \mathrm{CFI}=0.90, \mathrm{NNFI}=0.88$, and $\mathrm{IFI}=0.89, \mathrm{RMR}=0.065, \beta=0.58$ ), indicating that $\mathrm{H} 2, \mathrm{H} 3$, and $\mathrm{H} 4$ cannot be further tested. However, the RMSEA and 
NNFI values of Model I did not meet the recommended level of 0.08 and 0.90 for RMSEA and NNFI, respectively, which indicates that Model I may not be acceptable.

Model II included only the values factor of volunteer motivation when testing the full model. Although the $\chi^{2}$ value $\left(\chi^{2}=125.54, \mathrm{df}=51, \mathrm{p}<0.01\right)$ was significant, other fit indices achieved a marginally acceptable fit with the data (RMSEA $=0.085, \mathrm{CFI}=0.95, \mathrm{NNFI}=0.94$, $\mathrm{IFI}=0.92$, and $\mathrm{RMR}=0.068$ ). A $\chi^{2}$ difference test was then used to compare Model I to Model II. Model II (the full model) indicated significant difference in the fit for these two models, $\chi^{2}$ difference (32, $\mathrm{n}=223)=65.5, \mathrm{p}<0.001$, supporting the full model that included partial mediation.

However, Model III may not be acceptable because the RMSEA (0.086) and $\mathrm{RMR}(0.082)$ did not meet the cutoff of 0.08. Moreover, the $\chi^{2}$ difference test on the full model of Model II was significant, difference $(1, \mathrm{n}=217)=7.49, \mathrm{p}<0.01$, thereby supporting Model II. That is, value congruence did not completely mediate the relationship between values and organizational commitment, but partial mediation was evident, which indicates that the present study partially supports H1.

\section{Discussion}

Results from the three models offered support for a partial mediation effect of volunteer motivation on organizational commitment, suggesting that value congruence partially mediates the effects of values on organizational commitment. This indicates that although volunteer motivation influences the level of organizational commitment, volunteers who are motivated by their values would be more satisfied with their volunteer experience and consequently be more committed to the nonprofit sport organization.

The findings are consistent with previous organizational research in that volunteer motivation predicts organizational commitment if the volunteer's motivation is satisfied Based on Clary et al.'s work on functional motives for volunteering, it is suggested that workers initially engage in organizational citizenship behavior to satisfy particular functional needs. Individuals are then more likely to continue to volunteer if the experience as a whole is rewarding, satisfying. To be precise, the volunteer's satisfaction would contribute to a stronger sense of organizational commitment.

This study also contributes to the ongoing research on organizational commitment by testing a mediating variable. Value congruence plays a mediating role in many other relationships in the process of organizational commitment. Considering the results of previous studies, the present study suggests that greater attention to potential mediations among various relationships through using SEM may be useful for providing a more interpretable mechanism to analyze how and why a relationship exists between the predictor and dependent variable.

\section{References}

[1] Clary, E.G., Snyder, M., et al.,"Understanding and assessing the motivations of volunteers: a functional approach",Journal of Personality and Social Psychology, Vol. 74 No. 6, pp. 16-22, 1998. 